\title{
9 Einflüsse des Deutschen auf das Tschechische: Ein Sprachvergleich aus der Lernerperspektive
}

\subsection{Einführung}

Tschechisch gehört im Gegensatz zu Deutsch zur slawischen Sprachfamilie. Genauer gesagt bildet das Tschechische zusammen mit dem Polnischen, Slowakischen, Schlesischen, Kaschubischen und Nieder-/Obersorbischen die westslawische Sprachgruppe. Beide Kulturen haben jedoch jahrhundertelang aufeinander gewirkt ${ }^{130}$ und sich gegenseitig beeinflusst. Es ist daher nicht überraschend, dass auch die tschechische Sprache nicht unbeeinflusst geblieben ist. In einer früheren Studie zur Anwendung von lexikalischen Germanismen im modernen Tschechisch (Schmiedtová/Schmiedtová 1996) wurde anhand von korpusbelegtem ${ }^{131}$ Sprachmaterial gezeigt, dass Germanismen einen festen Status im Lexikon des Tschechischen haben und dass sie von tschechischen Muttersprachlern produktiv gebraucht werden.

Aus weiteren Arbeiten ist ersichtlich geworden, dass der Einfluss des deutschen auf das tschechische Sprachsystem nicht auf den lexikalischen Bereich beschränkt bleibt: Es zeigt sich (Schmiedtová 2003a, 2003b), dass verbale Präfixe im Deutschen und Tschechischen überraschend viele Gemeinsamkeiten aufweisen. Aber auch in Bezug auf die Verbstellung lassen sich in den zwei typologisch unterschiedlichen Sprachen bestimmte Ähnlichkeiten feststellen (Schmiedtová 2004).

Zusammenfassend kann man festhalten, dass der lange Sprach- und Kulturkontakt und die geografische Nähe zu Ähnlichkeiten in einigen sprachlichen Bereichen geführt hat. Der Einfluss des Deutschen geht über den Bereich des Lexikons hinaus und schlägt sich systematisch auch im Bereich der tschechischen Syntax nieder.

Der Fokus dieses Kapitels ${ }^{132}$ liegt nur indirekt auf dem Sprachvergleich zwischen dem Deutschen und Tschechischen. Das zentrale Thema stellt der Erwerb

130 Im Jahre 1526 bestiegen die Habsburger unter Ferdinand I. von Habsburg den böhmischen Thron und Böhmen wurde Teil der Habsburgermonarchie. Erst nach dem durch die Niederlage im Ersten Weltkrieg ausgelösten Fall der Österreichisch-Ungarischen Monarchie wurde am 28. Oktober 1918 die autonome Tschechoslowakische Republik gegründet.

131 Die Korpora, die für diese Studie verwendet wurden, sind alle Teil des Tschechischen Nationalkorpus (vgl. auch: http://ucnk.ff.cuni.cz/english/index.html).

132 Die Datenerhebung wäre nicht möglich gewesen ohne die Unterstützung des Goethe-Instituts in Prag. Ich möchte mich herzlich bei der damaligen Direktorin Frau Ute Gräfin Baudissin 
des Tschechischen als Fremdsprache dar, der in einer Studie zum Erwerb der temporalen Simultanität von Schmiedtová (2004) empirisch untersucht wurde. Das vorliegende Kapitel bezieht sich auf diese Studie.

In der Erwerbsforschung wird allgemein angenommen, dass typologische Nähe/Distanz eine wichtige Rolle beim Erwerb einer Fremdsprache spielt. Neben der Typologie spielt aber auch die so genannte Psychotypologie (Kellerman 1983a) eine wichtige Rolle.

The exact nature of what does constitute this subset will [...] depend not only on what I have called the learner's psychotypology but also on a second constraining factor, the transferablility of the L1 structure, that is, the probability with which this structure will be transferred relative to other structures in the L1. Transferability is to be seen as a theoretical notion, which derives from native speaker's own perception of the structure of their language. If a feature is perceived as infrequent, irregular, semantically or structurally opaque, or in any other way exceptional, what we could in other words call 'psycholinguistically marked', then its transferability will be inversely proportional to its degree of markedness. Transferability is not itself a predictor of performance but it is one of the determinants of whether an L1 structure will be treated as language-specific (not transferable to a given L2) or language-neutral (that is, transferable to a given L2). (ebd.: 117)

Die Psychotypologie ist insbesondere mit Blick auf den Transfer sprachlicher Merkmale aus dem System der Muttersprache in das der Zielsprache relevant. Der Begriff bezieht sich darauf, wie die allgemein angenommene typologische Verwandtschaft zweier Sprachen durch den Lernenden empfunden und wie dieses Empfinden im Prozess des Erwerbs praktisch umgesetzt wird. Mit anderen Worten, das implizite - wenn auch relativ oberflächliche - Wissen des Lerners über die Unterschiede bzw. die Ähnlichkeiten zwischen eigener Muttersprache und der zu lernenden Zielsprache beeinflussen, mit welchen Erwartungen und Haltungen der Lerner an den Erwerb der Zielsprache herangeht. So wird beispielsweise in einem Lehrbuch „Einstieg Tschechisch“ das Tschechische als „eine der schwersten Sprachen in Europa“ (Henßen/Sobkuljak 2005) bezeichnet. Diese Einschätzung hängt wahrscheinlich auch damit zusammen, dass das Tschechische - wie alle anderen slawischen Sprachen - obligatorisch den Aspekt zum Ausdruck bringt (siehe Kap. 2 und 5). Das aspektuelle System gilt für Sprecher von nicht-aspektuellen Sprachen wie dem Deutschen als kaum erlernbar (siehe Kap. 7).

bedanken. Sie hat es möglich gemacht, dass ich für die Dauer von drei Monaten in den Räumen des Goethe-Instituts Aufnahmen machen konnte. Mein Dank geht auch an Angelika Hájková und Monika Valter am Goethe-Institut Prag, die mich bei der Rekrutierung der Lerner und den logistischen Anforderungen des Projektes unterstützt haben. 
So behauptet Grekhova (1985), dass deutsche Lerner des Russischen niemals das aspektuelle System des Russischen beherrschen können. Wenn diese Behauptung wahr sein sollte, wären deutsche Lerner auch von einem erfolgreichen Erwerb des Tschechischen ausgeschlossen. Diese Behauptungen werden im späteren Verlauf des Kapitels aufgegriffen und an Hand von empirischen Daten überprüft.

Im Fokus dieses Kapitels steht eine umfangreiche Studie (Schmiedtová 2004), die sich mit dem Verlauf und dem Endzustand des Erwerbs des Tschechischen durch deutsche Muttersprachler beschäftigt. Wir werden in dieser Studie auf die Frage eingehen, wie deutsche Lerner bei dem Erwerb des Tschechischen vorgehen (Verlauf) und inwieweit sie sich dabei dem System der Zielsprache nähern können (Endzustand), d.h. wie erfolgreich sie beim Erwerb des Tschechischen tatsächlich sind. Um diesen beiden Fragestellungen besser nachgehen zu können, wird eine Gruppe von englischen Lernern des Tschechischen - die eine Muttersprache mit ausgebildetem Aspektsystem mitbringen - zum Vergleich herangezogen.

\subsection{Deutsche Lerner und englische Lerner des Tschechischen}

\subsubsection{Fragestellung}

Die Ausgangsfrage der diesem Kapitel zugrundeliegenden Studie lässt sich wie folgt umreißen: Wie gehen deutsche und englische Muttersprachler vor, wenn sie in ihrer L2 Tschechisch 'temporale Gleichzeitigkeit' ausdrücken? Der Begriff der temporalen Gleichzeitigkeit bezieht sich dabei auf eine Situation, in der zwei Ereignisse zur gleichen Zeit passieren und sich ganz oder zum Teil überlappen. So kann man sich beispielsweise eine Situation vorstellen, in der jemand ein Buch liest und eine andere Person zur gleichen Zeit einen Artikel schreibt (eine vollständige Überlappung). Natürlich ist auch eine andere Situation denkbar, in der jemand ein Buch liest, während eine andere Person in das Zimmer hereinkommt (ein zeitliches Einschließen eines Ereignisses in das andere). Es gibt weitere Möglichkeiten (Simultanitätstypen), wie zwei Ereignisse temporal angeordnet werden können, wenn sie gleichzeitig geschehen (Schmiedtová 2004: 10-15). Auf diese wird hier jedoch nicht näher eingegangen. 


\subsubsection{Methode der Datenerhebung und Probanden}

Bei der Erforschung eines beliebigen linguistischen Phänomens im Kontext des Fremdspracherwerbs ist es wichtig zu wissen, wie das zu erwerbende System der L2 aufgebaut ist und wie es funktioniert. Ebenso wichtig ist es, sich über das System der Ausgangssprache(n) Klarheit zu verschaffen. Diese Ausgangsbasis - die so genannte baseline - ist eine unentbehrliche Voraussetzung für die vergleichende empirische Sprachforschung. Als baseline können prinzipiell frühere Studien oder Beschreibungen des untersuchten Phänomens in einer gegebenen Sprache dienen. Im Fall der Erforschung der temporalen Simultanität ergeben sich mehrere Probleme. Simultanität und ihr sprachlicher Ausdruck sind so gut wie nicht untersucht worden. Die Studien, die zum Thema der Simultanität zu finden sind, beschränken sich auf den Erstspracherwerb und die Untersuchungssprache - vorwiegend Englisch (Schmiedtová 2004: 1-5). Für die Beschreibung des Deutschen und des Tschechischen liegen nach aktuellem Wissensstand überhaupt keine Arbeiten vor. Aus diesen Gründen mussten für die Studie eigene Produktionsdaten in der Muttersprache erhoben werden. Diese bildeten die baseline für die Untersuchung der lernersprachlichen Daten.

Als Methode der Datenerhebung diente die Elizitation. Bei diesem Verfahren werden mit Hilfe von experimentellem Material bestimmte sprachliche Konstruktionen oder Äußerungen gezielt produziert. Den Probanden wurden auf einem Monitor elf kurze Werbefilme präsentiert (Schmiedtová 2004), die zwei simultane Ereignisse zeigten. Um die dargebotene Szene treffend beschreiben zu können, musste das Simultane an der Szene zum Ausdruck gebracht werden. Nach jedem Werbeclip wurde die Darbietung unterbrochen, der Proband sollte in eigenen Worten beschreiben, was er gesehen hatte. Danach wurde das Experiment fortgesetzt. Wenn ein Proband Interpretationsprobleme hatte, konnten die Clips auch mehrfach angesehen werden. Die Verbalisierungen der Probanden wurden mit einem digitalen Kassettenrecorder aufgenommen.

Bei der Datenerhebung der Lerner wurde zusätzlich auf die Reihenfolge der verwendeten Sprachen geachtet (Muttersprache und Zielsprache): Die Hälfte der Lerner führte die Aufgabe in der Reihenfolge 'Muttersprache vor Zielsprache' durch. Bei der anderen Hälfte der Lerner war die Abfolge umgekehrt: Zielsprache vor Muttersprache. Die spätere statistische Auswertung zeigte jedoch, dass die Reihenfolge der Sprachen keine Transfer-Effekte bewirkt hatte (Schmiedtová 2004: 221).

Für die Untersuchung wurden muttersprachliche L1-Daten erhoben. Dafür wurden insgesamt 60 Probanden aufgenommen, die drei Gruppen mit jeweils zwanzig deutschen, englischen und tschechischen Muttersprachlern bildeten. Deutsche und englische Muttersprachler wurden darüber hinaus (mit je 20 Ler- 
nern pro Gruppe) als Lerner des Tschechischen aufgenommen. Die Übersicht über die Probandenzahlen pro Gruppe lässt sich Tabelle 16 entnehmen. Die Abkürzung ZS steht für Zielsprache, die Abkürzung AS steht für Ausgangssprache.

Tab. 16: Überblick über Probanden

\begin{tabular}{llll}
\hline & Tschechisch (ZS) & Deutsch (AS) & Englisch (AS) \\
\hline Tschechisch (ZS) & 20 & - & - \\
\hline Deutsch (AS) & 20 & 20 & - \\
\hline Englisch (AS) & 20 & - & 20 \\
\hline
\end{tabular}

Alle Probanden füllten einen Fragebogen aus, in dem biografische Daten und soziale Variablen ermittelt wurden. Dabei wurden die sozialen Variablen Alter und Bildung bei den Versuchspersonen kontrolliert. So wurden für das Experiment Erwachsene im Alter von 25 bis 40 Jahren ausgewählt, die ihre Muttersprache in einer monolingualen Umgebung erworben hatten. Der Erwerb aller Fremdsprachen (inklusive des Tschechischen) erfolgte bei allen Probanden erst nach dem abgeschlossenen Erstspracherwerb.

Alle Versuchspersonen waren entweder Studierende oder Mitarbeiter der Karlsuniversität oder des Goethe-Institutes in Prag. Das Durchschnittsalter für deutsche Muttersprachler/Lerner war 37,6 Jahre, für englische Muttersprachler/ Lerner 34,2 Jahre und für tschechische Muttersprachler 32,9 Jahre. Alle Daten wurden in Prag erhoben. Die Geschlechterverteilung war nur bei den tschechischen Probanden gleichmäßig. In beiden Lernergruppen waren mehr Versuchspersonen männlich als weiblich: 65\% Männer, 35\% Frauen.

Auf diese Weise wurden Sprachproduktionsdaten von insgesamt 60 Muttersprachlern und 40 Lernern erhoben. Die Kontrolle über relevante Einflussfaktoren für Spracherwerb und Sprachverwendung stellte sicher, dass vergleichsweise homogene Sprechergruppen dem Vergleich zu Grunde gelegt werden konnten.

\subsubsection{Die untersuchten Sprachen}

Der folgende Abschnitt befasst sich mit dem jeweiligen Sprachsystem der drei untersuchten Sprachen. Wie in Abschnitt 9.1 erwähnt, gehören das Deutsche und das Tschechische zu unterschiedlichen Sprachfamilien. Das Englische - als die sprecherreichste germanische Sprache - fällt mit dem Deutschen in die Gruppe der germanischen Sprachen. 
In Bezug auf die zentrale Fragestellung von Schmiedtová (2004) ist es wichtig zu betonen, dass das Konzept der Simultanität in allen untersuchten Sprachen zum Ausdruck gebracht werden kann. Der Unterschied besteht jedoch darin, mit welchen sprachlichen Mitteln dieses Konzept im Deutschen, Englischen und Tschechischen ausgedrückt wird. Bevor über die wichtigsten Ergebnisse der Studie von 2004 berichtet wird, sollen zuerst die verschiedenen sprachlichen Mittel, die den Sprechern in den jeweiligen Sprachen zum Ausdruck der Simultanität zur Verfügung stehen, betrachtet werden.

Die zwei wichtigsten Mittel sind a) grammatische Kategorien der Verben, z.B. Tempus und insbesondere Aspekt, und b) verschiedene Typen von temporalen Adverbien, einschließlich temporaler Subordinationen. Das Spektrum der temporalen Adverbien ist in allen drei Sprachen vergleichbar. Die markantesten Unterschiede lassen sich in der Art und Weise festmachen, wie die drei untersuchten Sprachen aspektuelle Relationen ausdrücken (siehe Abb. 32 und Kap. 2, 5-7).

Ausgangssprache 1

\section{Englisch \\ Aspekt ist grammatikalisiert \\ - Progressiv (-ing)}

Ausgangssprache 2

\section{Deutsch}

Aspekt ist nicht grammatikalisiert

Tschechisch

Aspekt ist grammatikalisiert

- Perfektiv/Imperfektiv

(verbale Präfixe, Suffixe)

Abb. 32: Übersicht über die drei untersuchten Sprachen

Aus Abbildung 32 ergibt sich, dass bezüglich des \pm Vorhandenseins der Kategorie des grammatischen Aspekts in den Sprachsystemen der drei Sprachen das Englische mit dem Tschechischen eine Gruppe bildet. Mit anderen Worten, trotz typologischer Nähe zum Deutschen steht das Englische im Bereich des Aspekts dem Tschechischen näher als dem Deutschen.

Anhand dieser relativ oberflächlichen Gegenüberstellung der drei Sprachen, die sich lediglich auf die üblichen grammatischen Beschreibungen stützt, lassen sich die ersten Hypothesen hinsichtlich der Lerner formulieren. 


\section{Hypothese 1}

Da der Ausdruck der Simultanität eng mit der Aspektmarkierung zusammenhängt, könnte man annehmen, dass englische Lerner des Tschechischen von der Ähnlichkeit des Ausgangs- und des Zielsystems profitieren. Sie werden schnell in der Lage sein, die Ähnlichkeiten zwischen ihrer Muttersprache und der Zielsprache zu identifizieren. Dann werden sie die sich ähnelnden Merkmale aus ihrer L1 in die L2 übertragen. Man spricht in diesem Fall auch vom positiven Transfer.

\section{Hypothese 2}

Deutsche Lerner dagegen werden große Lernschwierigkeiten beim Erwerb der aspektuellen Kategorien in L2 Tschechisch haben. Wenn sich dies bestätigt, dann werden sie sich auch schwertun, wenn sie in L2 Tschechisch Simultanität zum Ausdruck bringen müssen. Sie werden nicht zielsprachig vorgehen (können), sondern auf andere Strategien zurückgreifen.

Im Folgenden werden diese Hypothesen anhand von empirischen Daten überprüft und zum Teil auch in Frage gestellt. Zunächst werden die Produktionsdaten der Muttersprachler gesichtet und miteinander verglichen. Im Anschluss daran wird der Fokus auf den Lernerdaten liegen. Dabei werden erneut die Ausgangsfragestellungen aufgegriffen: Wie gehen deutsche Lerner im Erwerb der Fremdsprache Tschechisch vor (Verlauf) und inwieweit können sie die Zielsprache in bestimmten Bereichen erlernen (Endzustand)? Zu diesem Zweck wird die deutsche mit der englischen Lernergruppe des Tschechischen verglichen.

\subsubsection{Die Ergebnisse: Muttersprachler}

Die Studie ergab folgende Präferenzen für die Muttersprachler der drei untersuchten Sprachen: Sprecher des Tschechischen und Englischen ziehen es vor, primär aspektuelle Mittel in Kombination mit lexikalischen Mitteln zum Ausdruck der Simultanität zu verwenden. Es ist hier wichtig zu betonen, dass diese Präferenzen nicht etwa eine zufällige und subjektive Wahl der Sprecher widerspiegeln, sondern auf den in der Grammatik der jeweiligen Sprache verankerten Strategien beruhen.

Die von tschechischen und englischsprachigen Probanden verwendete Strategie wird am Beispiel (63), das von einem tschechischen Muttersprachler stammt, veranschaulicht.

Tak on to slizává (Impf.Präs), někdo otevře (Perf.Präs) dveře 'So, er ist dabei es abzulecken, jemand öffnet die Tür' 
Wie Beispiel (63) zu entnehmen ist, ergibt sich aus dem Gebrauch von zwei mit verschiedenen Aspekten markierten Verben ablecken und öffnen eine simultane Interpretation der Gesamtäußerung. ${ }^{133}$ Mit anderen Worten, die Ereignisse 'jemand leckt etwas ab' und 'ein anderer öffnet die Tür' müssen sich zeitlich überlappen. Ähnliche Beispiele finden wir auch in den Daten der englischen Muttersprachler.

... and he took (Perf.Verg) a sip from his juice, she was coming (Impf.Verg) back

'... und er nahm einen Schluck Saft, sie war zurückgekommen'

Diese auf aspektuellen Mitteln beruhende Strategie, die als the stonger aspectual style identifiziert und bezeichnet wird (Schmiedtová 2004: 135), lässt sich mit zusätzlichen lexikalischen Mitteln, wie z.B. temporalen Adverbien, ergänzen. So ergibt sich der so genannte weaker aspectual style ${ }^{134}$ (Schmiedtová 2004: 135). Dieser wird in Beispiel (65) veranschaulicht.

(65) A začne tu hořčiči slízávat (Impf.Präs) a v tom (TAdv) přijde (Perf.Präs) jeho starši brácha

'Und er fängt an den Senf abzulecken und in dem Moment kommt sein älterer Bruder herein'

Die schwächere Aspekstrategie wird nicht nur von tschechischen, sondern häufig auch von englischen Muttersprachlern verwendet. Dagegen wählen die deutschen Sprecher, wenn sie in ihrer Muttersprache über simultane Ereignisse sprechen, die adverbiale Strategie (the adverbial style, Schmiedtová 2004: 135). Durch diese Strategie wird die Simultanität mittels verschiedener temporaler Adverbien zum Ausdruck gebracht. Dabei spielt die aspektuelle Markierung keine Rolle. Typisch für das Deutsche ist die in Beispiel (66) angeführte Versprachlichung, die von einem deutschen Muttersprachler stammt.

133 Der Begriff Gesamtäußerung bezieht sich auf die Verbalisierung des simultanen Ereignisses. Eine Gesamtäußerung besteht aus mehreren (mindestens zwei) Teiläußerungen.

134 Die schwächere aspektuelle Strategie beruht auf der Kombination von lexikalischen (temporale Adverbien) und aspektuellen Mitteln. Im Gegensatz dazu wird bei der stärkeren aspektuellen Strategie nur der Aspekt gebraucht, um Simultanität auszudrücken. Aus dieser Gegenüberstellung ergeben sich die Bezeichnungen 'stärker' und 'schwächer'. 
In diesem Moment (TAdv) kommt vielleicht der ältere Bruder herein

An dieser Stelle ist anzumerken, dass auch die englischen und tschechischen Muttersprachler die adverbiale Strategie verwenden können. Sie tun es aber viel seltener als die Deutschen und gebrauchen dabei Verben, die entweder keine explizite Aspektmarkierung aufweisen, oder Verben, die explizit nur für den perfektiven Aspekt markiert sind. Wenn das Erste zutrifft, kommen im Tschechischen die so genannten Simplex-Formen vor (Schmiedtová 2004), z.B. dát ('geben') oder psát ('schreiben'), während das Englische sich der unmarkierten simple present- oder simple past-Formen, aber nicht der entsprechenden progressive-Formen (Markierer -ing) bedient (siehe Kap. 2 und 8).

Da nur im Tschechischen und nicht im Englischen eine systematische Markierung des perfektiven Aspekts zur Verfügung steht (Schmiedtová 2004; Kap. 2), kommen zwei aufeinander folgende perfektiv markierte Verben in der adverbialen Strategie nur im Tschechischen vor. In diesem Fall hebt die Präsenz eines temporalen Adverbs die kanonische Interpretation einer temporalen Sequenz auf. Diese kanonische sequenzielle Interpretation wird in (67) gezeigt. Beispiel (68) veranschaulicht die gleiche aspektuelle Kombination wie in (67) mit einem zusätzlichen temporalen Adverb. Die Äußerungen in (68) werden als simultan interpretiert.

(67) Přistoupí (Perf.Präs) k plakátu, olízne (Perf.Präs) kečup a hořčici 'Er tritt zum Poster, (er) leckt den Ketchup und den Senf ab'

(68) Přistoupí (Perf.Präs) k plakátu, přičemž (TAdv) olizne (Perf.Präs) kečup a hořčici

'Er tritt zum Poster, während (er) den Ketchup und den Senf ableckt'

Der Gebrauch der einzelnen Strategien ist von den Sprechern des L1 Tschechischen, L1 Englischen und L1 Deutschen in Tabelle 17 zusammengefasst. Die Prozentwerte geben an, wie häufig eine bestimmte Vorgehensweise in Relation zu der Gesamtzahl der produzierten Äußerungen (n) eingesetzt wurde (Tschechisch $\mathrm{n}=83$, Englisch $\mathrm{n}=87$, Deutsch $\mathrm{n}=81$ ). 
Tab. 17: Die Präferenzen der Muttersprachler für den Simultanitätsausdruck in ihrer jeweiligen Muttersprache (L1) (* weist auf einen statistisch signifikanten Unterschied hin)

\begin{tabular}{llcc}
\hline & Tschechisch L1 & Englisch L1 & Deutsch L1 \\
\hline stärkere Aspektstrategie & $18 \% \star$ & $8 \%$ & $0 \%$ \\
\hline schwächere Aspektstrategie & $71 \%$ & $76 \%$ & $0 \%$ \\
\hline adverbiale Strategie & $11 \%$ & $16 \%$ & $100 \%$ \\
\hline
\end{tabular}

Anhand von Tabelle 17 kann das folgende Fazit gezogen werden: Die Präferenzen oder die Muster, denen die tschechischen und englischen Muttersprachler in ihrer Muttersprache folgen, weisen starke Ähnlichkeiten auf. Der einzige signifikante Unterschied, der sich bei der statistischen Analyse der Produktionsdaten ergab, bezieht sich auf die Häufigkeit des Gebrauchs des stronger aspectual style. Diese Strategie wird im Tschechischen häufiger als im Englischen in Anspruch genommen $(z=1,75, p<.05)$.

Diese Ergebnisse werden in Schmiedtová (2004: 169-174) so interpretiert, dass der Einsatz der aspektuellen Mittel für den Ausdruck der Simultanität im Tschechischen und Englischen nahezu obligatorisch ist: Die Häufigkeit, mit der die stärkere und schwächere Aspektstrategie angewendet wird, erreicht $89 \%$ für das Tschechische bzw. 84\% für das Englische. Zudem kommt die stärkere Strategie im Tschechischen signifikant öfter vor als im Englischen. Dieses Resultat deutet daraufhin, dass die explizite Aspektmarkierung, die am Verb mit Hilfe von Präfixen und Suffixen zum Tragen kommt, den grundlegenden Ausdruck für Simultanität darstellt. Das unterstreicht die essenzielle Rolle des Aspekts im Sprachsystem des Tschechischen.

Im Vergleich zu den tschechischen und englischen Sprechern geht die Lerngruppe der deutschen Muttersprachler durchgängig anders vor. Den deutschen Sprechern steht kein grammatischer Aspekt in ihrer Muttersprache zur Verfügung und sie greifen deswegen auf diverse lexikalische Mittel zurück, insbesondere auf Adverbien. Diesem Muster folgten ohne Ausnahme alle Sprecher, die an dieser Studie teilgenommen haben. Die adverbiale Strategie ist die Strategie der deutschen Sprecher.

\subsubsection{Die Ergebnisse: Deutsche Lerner des Tschechischen}

Auf den ersten Blick gehen beide Lernergruppen (20 Versuchspersonen pro Lernergruppe) beim Ausdruck der Simultanität im L2 Tschechisch so vor, dass sie 
das dominante Muster ihrer Muttersprache in die Zielsprache übertragen. Dementsprechend verwenden deutsche Lerner im Tschechischen die adverbiale Strategie zu 50\% und verzichten dabei auf die explizite Aspektmarkierung, während die englischen Lerner eine der zwei Aspektstrategien mit einer Häufigkeit von 90\% benutzen und sich so auf die explizite Aspektmarkierung stützen. Eine solche sprachliche Darstellung der Stimuli ist zwar nicht falsch, aber viel zu ungenau.

Der Grund dafür ist, dass die verschiedenen Sprachniveaus ${ }^{135}$ der Lerner hier nicht miteinbezogen sind. Bei Berücksichtigung dieser ergibt sich ein viel detaillierteres Bild, welches mehr Informationen über den Verlauf und den Endzustand des L2-Erwerbs vermittelt.

Zuerst stehen die Vorgehensweisen beim Ausdruck von Simultanität in L2 Tschechisch unter Berücksichtigung der verschiedenen Sprachniveaus der deutschen Lerngruppe im Vordergrund. Die eingesetzten Strategien sind in Tabelle 18 zusammengefasst und dem zielsprachigen Muster gegenübergestellt.

Tab. 18: Der Ausdruck der Simultanität von deutschen Lernern unter Berücksichtigung des Sprachniveaus

\begin{tabular}{lclll}
\hline deutsche Lerner & $\begin{array}{l}\text { Anfänger } \\
(\mathbf{N}=9)\end{array}$ & $\begin{array}{l}\text { Zwischenstufe } \\
(\mathbf{N}=9)\end{array}$ & $\begin{array}{l}\text { Fortgeschritten } \\
(\mathbf{N}=8)\end{array}$ & $\begin{array}{l}\text { tschechische } \\
\text { Muttersprachler }\end{array}$ \\
\hline $\begin{array}{lccl}\text { stärkere Aspektstrategie } \\
\text { schwächere Aspekt- }\end{array}$ & $0 \%$ & $25 \%$ & $13 \%$ & $18 \%$ \\
$\begin{array}{l}\text { strategie } \\
\text { adverbiale Strategie }\end{array}$ & $100 \%$ & $25 \%$ & $42 \%$ & $71 \%$ \\
\hline
\end{tabular}

Tabelle 18 zeigt, dass deutschsprachige Anfänger ausschließlich die adverbiale Strategie verwenden, wenn sie in L2 Tschechisch über simultane Ereignisse sprechen (100\%, 18 Äußerungen). Da dies die Hauptstrategie für den Simultanitätsausdruck deutscher Muttersprachler im Deutschen ist, lässt sich annehmen, dass deutsche Lerner sich zu Beginn des Erwerbs der Zielsprache auf die aus der Muttersprache bekannten Strategien stützen. Zur Veranschaulichung dient das folgende Beispiel, das von einem deutschen Anfänger des Tschechischen aus der

135 Die Ermittlung der Sprachniveaus ist sehr schwierig, und es herrscht in der einschlägigen Literatur kein Konsens darüber, wie dabei vorgegangen werden soll. Die Methode, mit der das Sprachniveau der Lerner in dieser Studie ermittelt wurde, ist im Detail in Schmiedtová (2004: 107-121) beschrieben (siehe auch Kap. 1, Abschn. 1.3). 
Studie stammt. Um die Authentizität zu bewahren, wird dieses Beispiel nicht korrigiert wiedergegeben, sondern in der Form gelassen, in der es produziert wurde.

Ten, $v$ ten moment (TAdv) otec tam ten kluk, on ji (Impf.Präs) sendvič 'Der, in dem Moment, Vater dort, dieser Junge, er isst den Sandwich'

Die erste Äußerung in Beispiel (69) enthält zwar kein Verb, dafür aber ein temporales Adverb: $v$ ten moment ('in diesem Moment'). Durch das Adverb wird signalisiert, dass die Gesamtäußerung simultan interpretiert wird. Diese Interpretation würde auch dann gelten, wenn das in der nächsten Teiläußerung auftretende Verb eine explizite aspektuelle - perfektive oder imperfektive -Markierung tragen würde.

Die nächste Gruppe bilden Lerner, die sich im Erwerb des Tschechischen auf der Zwischenstufe befinden. Tabelle 18 zeigt, dass Lerner dieser Gruppe insofern fortgeschrittener sind, als dass sie im Tschechischen verschiedene Strategien anwenden, um temporale Simultanität zum Ausdruck zu bringen. Ähnlich wie die Anfänger verwenden sie noch häufig die adverbiale Strategie (50\%, 20 Äußerungen), woraus zu schließen ist, dass dieser Strategie nach wie vor eine besondere Stellung in der Lernergrammatik zukommt. Hier ein Beispiel aus dem Lernerkorpus:

Takže vešel (Perf.Verg) do prostoru

a s jazykem to zkusil (Perf.Verg) to udělat pryč

a v tomto momentu (TAdv) ten spíš ten, ten starší bratr přišel (Perf.Verg)

'So ist er in den Raum hineingegangen und er versuchte mit der Zunge es wegzumachen und in diesem Moment kam wahrscheinlich der ältere Bruder hinein rein'

Worin besteht der Unterschied zwischen den Anfängern und den Lernern, die sich auf der Zwischenstufe befinden? Bezüglich des Gebrauchs der adverbialen Strategie hat die statistische Analyse keine Signifikanzen gezeigt $(z=0,56, n s)$. Wenn man den Gebrauch der anderen beiden Strategien auf diesem Sprachniveau betrachtet, sieht man, dass Lerner den Aspekt durchaus anwenden, wenn Simultanität in der Zielsprache ausgedrückt wird: Die stärkere aspektuelle Strategie wird in 25\% (10 Äußerungen) der Fälle gebraucht. Dieselbe Verteilung gilt auch für die schwächere aspektuelle Strategie: 25\%, 10 Äußerungen. Der Anstieg im Gebrauch der expliziten aspektuellen Markierung, die für die beiden aspektuellen Strategien nötig ist, deutet auf einen kritischen Entwicklungsschritt hin. Auf diesen Punkt wird in der abschließenden Diskussion im Abschnitt 9.3 näher eingegangen.

Auch die fortgeschrittenen deutschen Lerner gebrauchen die adverbiale Strategie, allerdings weniger häufig (45\%, 18 Äußerungen) als die Lerner auf der Zwi- 
schenstufe. Diese Differenz ist statistisch jedoch nicht signifikant $(\mathrm{z}=0,48, n s)$. Der Unterschied zu der vorherigen Erwerbsstufe besteht in der signifikanten Verminderung des Gebrauchs der stärkeren aspektuellen Strategie $(z=1,68, p<.05)$. Bemerkenswert ist auch, dass die Häufigkeit der Anwendung der schwächeren aspektuellen Strategie mit der der adverbialen Strategie vergleichbar ist. Die schwächere Aspektstrategie kommt erstaunlich häufig vor: 42\%, 17 Äußerungen. Der Gebrauch dieser Strategie bei der fortgeschrittenen Lerngruppe ist signifikant höher als bei den Lernern auf der Zwischenstufe $(z=2,00, p<.05)$. Das nächste Beispiel (71) soll einen Eindruck vermitteln, wie ein fortgeschrittener deutscher Lerner des Tschechischen mit Hilfe der schwächeren aspektuellen Strategie eine simultane Situation beschreibt.

(71) a vezme (Perf.Präs) si nějaký nápoj, double cooler se jmenuje, $a v$ tý chvíli (TAdv) stojí (Impf.Präs) dole holka, je před nebo pod oknem a mává mu 'und (er) nimmt sich ein Getränk, es heißt double cooler, und in diesem Moment steht ein Mädchen unten, (sie) ist vor oder unter dem Fenster und winkt ihm zu'

Die Entwicklung, die vom Beginn bis zum Endzustand eines Erwerbsprozesses stattfindet, ist in Abbildung 33 veranschaulicht. Sie stellt die Präferenzen der deutschen Muttersprachler in ihrer L1 (German Native) dar, das bevorzugte Muster der tschechischen Muttersprachler (Czech Native) und schließlich auch die verschiedenen Strategien, die deutsche Lerner (German Basic, German Intermediate, German Advanced) in L2 Tschechisch anwenden.

Hier stellen sich gleich zwei Fragen: 1) Warum verwenden deutsche Lerner auf der Zwischenstufe neben der schwächeren aspektuellen Strategie (25\%) auch die stärkere aspektuelle Strategie (25\%)? 2) Warum reduziert sich die stärkere aspektuelle Strategie bei den fortgeschrittenen deutschen Lernern des Tschechischen von $25 \%$ auf $13 \%$ ?

Diese Unterschiede lassen sich so erklären, dass auf der Zwischenstufe die deutsche Lerngruppe den grammatischen Aspekt gewissermaßen 'entdeckt' und mit der in der Zielsprache sehr produktiven Markierung 'experimentiert'. In dieser kritischen Lernphase kommt es auf diese Weise zu einem übermäßigen Gebrauch der aspektuellen Markierung. Dieser schlägt sich auch in der ansteigenden Verwendung der stärkeren aspektuellen Strategie (ohne Verwendung der temporalen Adverbien) nieder.

Für die gleiche Entwicklungsstufe konnte ein übermäßiger Gebrauch auch für die Verwendung des mit Hilfe der Präfigierung gebildeten perfektiven Aspekts gezeigt werden (Schmiedtová 2004: 230-238): Deutsche Lerner auf der Zwischen- 
stufe bildeten eine große Anzahl von präfigierten Verben, die eine perfektive aspektuelle Bedeutung hatten. Dabei kombinierten sie die korrekte Form eines Präfixes mit einem Verb, das mit diesem Präfix in der Zielsprache nicht kombinierbar ist.

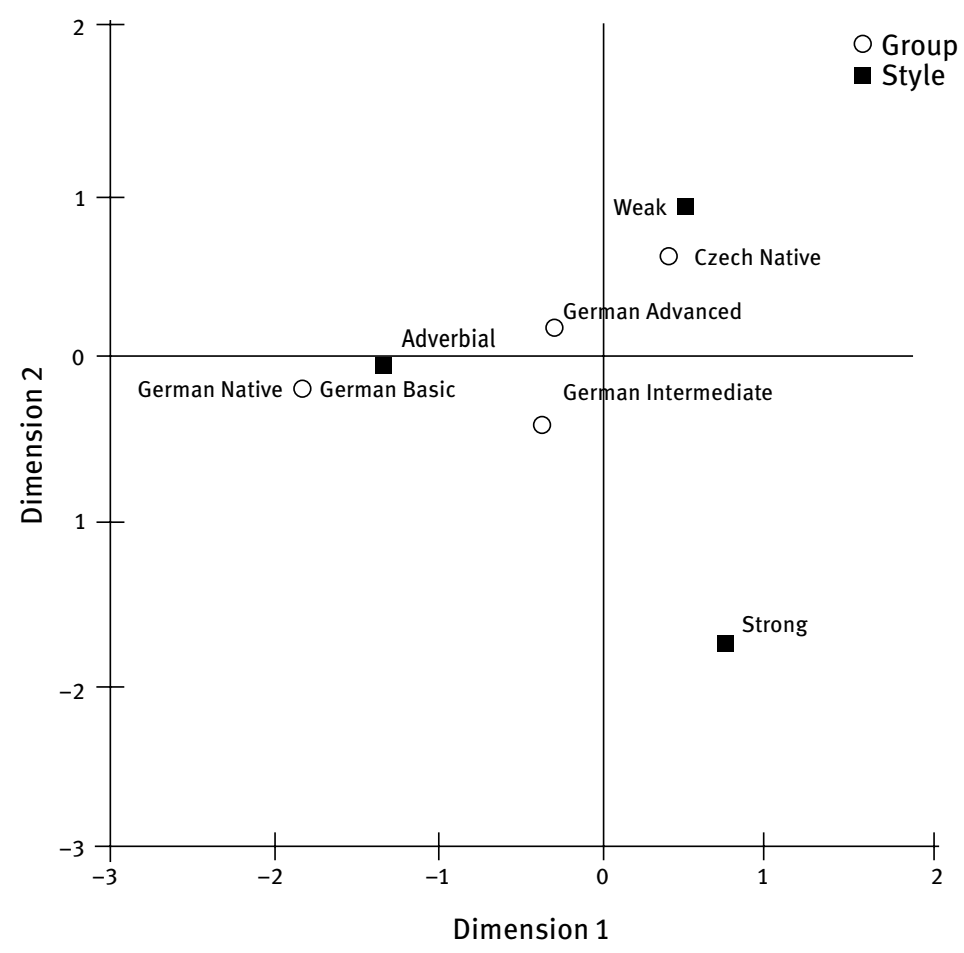

Abb. 33: Die so genannte 'centroids'-Darstellung ${ }^{136}$ für den Zusammenhang zwischen der verwendeten Strategie und den Sprechergruppen Tschechisch L1, Deutsch L1 und deutschen Lernergruppen L2 Tschechisch

Wie lässt sich Abbildung 33 interpretieren? Klar ist, dass deutsche Lerner zu Beginn des Erwerbs des Tschechischen so wie in ihrer Muttersprache vorgehen: Sie gebrauchen ausschließlich adverbiale Mittel, wenn sie Simultanität ausdrücken wollen. Auf der nächsten Stufe, der Zwischenstufe, wird viel häufiger der Aspekt gebraucht. Dieses fortgeschrittene Stadium bringt eine weitere Differen-

136 Die 'centroids'-Darstellung ist eine statistische Visualisierungsmethode für komplexere Daten. Als Grundlage werden alle Datenwerte einer Gruppe und nicht nur Durchschnittswerte verwendet. 
zierung im Bereich Aspekt mit sich: eine starke Präferenz zugunsten der schwächeren Aspektstrategie.

Deutsche Lerner können das Tschechische zu Beginn nur mit Hilfe der 'deutschen Strategien' erlernen. Sie transferieren das deutsche Muster, das auf dem Gebrauch der Adverbien beruht, ins Tschechische. Aber bereits auf der Zwischenstufe lernen sie die verbalen Aspektformen und setzen sie zielsprachig zum Ausdruck von Simultanität ein. Festzuhalten ist damit zunächst, dass deutsche Lerner den slawischen Aspekt erlernen und - zumindest in bestimmten Kontexten - zielsprachig korrekt anwenden können.

Dieses Ergebnis widerspricht der Studie von Grekhova (1985), in der die These vertreten wird, dass deutsche Lerner das aspektuelle System des Russischen nie lernen können. Zwar bezieht sich die Untersuchung auf das Russische, aber Tschechisch und Russisch sind in Bezug auf das Aspektsystem durchaus vergleichbar. Die Ergebnisse der vorliegenden Studie zeigen, dass das Tschechische als Aspekt-Sprache durchaus - wenn auch sehr schwer - erlernbar ist. Das gilt auch für Lerner, deren Muttersprache eine Nicht-Aspektsprache ist.

An dieser Stelle stellt sich die Frage, wie weit deutsche Lerner in ihrem Erwerb des Tschechischen kommen können? Um die Frage nach dem Endzustand beantworten zu können, müssen zuerst die Daten der englischen Lerner gesichtet werden. Anschließend werden die deutschen Lerner mit den englischen Lernern verglichen.

\subsubsection{Die Ergebnisse: Englische Lerner des Tschechischen}

Der allgemeine Entwicklungstrend, der in den Daten der englischen Lerngruppe $\mathrm{zu}$ beobachten ist, geht von einem dominanten Gebrauch der stärkeren im Vergleich zur schwächeren aspektuellen Strategie aus. Zur Veranschaulichung dient Tabelle 19.

Tab. 19: Der Ausdruck der Simultanität von englischen Lernern unter Berücksichtigung des Sprachniveaus

\begin{tabular}{lllll}
\hline englische Lerner & $\begin{array}{l}\text { Anfänger } \\
(\mathrm{N}=10)\end{array}$ & $\begin{array}{l}\text { Zwischenstufe } \\
(\mathrm{N}=7)\end{array}$ & $\begin{array}{l}\text { Fortgeschritten } \\
(\mathrm{N}=9)\end{array}$ & $\begin{array}{l}\text { tschechische } \\
\text { Muttersprachler }\end{array}$ \\
\hline stärkere Aspektstrategie & $90 \%$ & $40 \%$ & $33 \%$ & $18 \%$ \\
\hline $\begin{array}{l}\text { schwächere Aspekt- } \\
\text { strategie }\end{array}$ & $10 \%$ & $42 \%$ & $67 \%$ & $71 \%$ \\
\hline \begin{tabular}{l} 
adverbiale Strategie \\
\hline
\end{tabular} & $0 \%$ & $18 \%$ & $0 \%$ & $11 \%$ \\
\hline
\end{tabular}


Wie Tabelle $19 \mathrm{zu}$ entnehmen ist, verwenden die englischen Lerner von Anfang an die wesentlichen Ausdrucksmittel der Zielsprache im Kontext simultaner Ereignisse. Aspektuelle Morphologie wird auch von Anfängern, die sich zum Zeitpunkt der Aufnahme gerade drei Monate in Tschechien aufhielten, angewendet. Die Bildung der Formen ist nicht immer zielsprachig, aber die Formen lassen sich im gegebenen Kontext interpretieren. Dieses Resultat steht im Gegensatz zu früheren Studien (vgl. Hendriks 1999), die annehmen, dass englische Lerner anfangs ausschließlich temporale Adverbien als eine fallback strategy verwenden, um Simultanität in der Zielsprache auszudrücken.

Lerner auf der Zwischenstufe verwenden die anspruchsvollere kombinatorische Strategie - die schwächere aspektuelle Strategie. Zusätzlich wird die adverbiale Strategie eingesetzt. Auffällig ist, dass fortgeschrittene englische Lerner ausschließlich die zwei aspektuellen Strategien verwenden und ganz auf den Gebrauch der adverbialen Strategie verzichten.

Im Vergleich zu der Verteilung der drei Strategien, die die tschechischen Muttersprachler in ihrer Muttersprache anwenden (siehe Tab. 19), zeigen die englischen Lerner eine starke Tendenz, die beiden aspektuellen Strategien viel zu häufig zu gebrauchen. Das trifft vor allem auf die stärkere Aspektstrategie zu, die auf allen Erwerbsstufen von der englischen Lerngruppe in hohem Maße verwendet wird.

An dieser Stelle ist zu betonen, dass der übermäßige Gebrauch der stärkeren aspektuellen Strategie nicht ungrammatisch ist. Die mit Hilfe dieser Strategie geformten Versprachlichungen drücken Simultanität durchaus aus. Trotzdem ist diese Strategie viel mehr mit einer Lernerstrategie assoziiert, da sie von Muttersprachlern nicht nur selten benutzt wird, sondern auch in bestimmten Kontexten unangemessen ist. Mit anderen Worten, die englischen Lerner weichen durchgängig von der Zielsprache ab, indem sie der Distribution der einzelnen Strategien und somit auch den Präferenzen der tschechischen Muttersprachler nicht folgen bzw. sich im Erwerbsverlauf der zielsprachigen Verteilung nicht wirklich nähern. Ob ein Adverb in der Zielsprache verwendet oder nicht verwendet wird, ist keine Frage der subjektiven Wahl, sondern ergibt sich aus den spezifischen temporalen Verhältnissen.

Ähnlich wie in Abbildung 33 für deutsche Lerner zeigt Abbildung 34 die Präferenzen der englischen Muttersprachler in ihrer L1 (English Native) sowie das bevorzugte Muster der tschechischen Muttersprachler (Czech Native) und schließlich auch die verschiedenen Strategien, die englische Lerner (English Basic, English Intermediate, English Advanced) in L2 Tschechisch anwenden. 


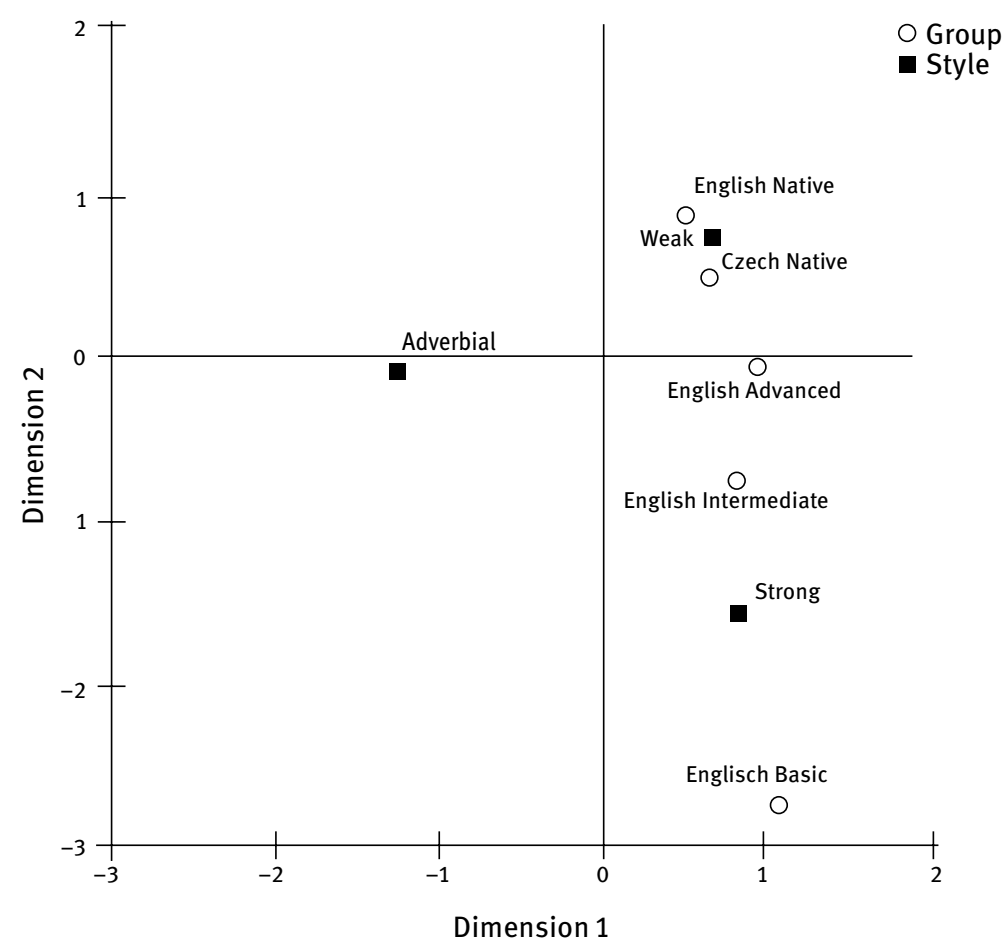

Abb. 34: Die so genannte 'centroids'-Darstellung für den Zusammenhang zwischen der verwendeten Strategie und der Sprechergruppen Tschechisch L1, Deutsch L1 und den englischen Lernergruppen L2 Tschechisch

Abbildung 34 zeigt, dass alle englischen Lernergruppen eine ausgeprägte Präferenz für die stärkere aspektuelle Strategie haben. Sie bevorzugen diese Strategie häufiger, als es die tschechischen und englischen Muttersprachler tun. Wie schon erwähnt, ermöglicht die Überlappung zwischen dem aspektuellen System des Englischen und des Tschechischen zu Beginn des Erwerbs Transfer-Strategien. Dementsprechend sind englische Anfänger in der Lage, die aspektuelle Markierung als grammatische Kategorie aus L1 Englisch für den Ausdruck der Simultanität in L2 Tschechisch zu übertragen.

Erstaunlich ist aber, dass die nicht-zielsprachige Präferenz für die stärkere Aspektstrategie im Laufe des Erwerbs von den englischen Lernern nicht in Richtung auf die zielsprachige Verteilung geändert wird. Die englischen Lerner bauen 
ihr L2-System graduell aus, indem sie auf der Zwischenstufe die schwächere aspektuelle Strategie und begrenzt auch die adverbiale Strategie in ihr Repertoire aufnehmen. Die adverbiale Strategie fällt aber bei den fortgeschrittenen Lernern ganz weg und die stärkere aspektuelle Strategie wird auch von den sehr fortgeschrittenen Lernern mit sehr guten Tschechisch-Kenntnissen fast doppelt so häufig wie von tschechischen Muttersprachlern gebraucht.

\subsubsection{Deutsche und englische Lerner im Vergleich}

Die Daten aus Schmiedtová (2004) haben gezeigt, dass englische Lerner schon am Anfang des Erwerbs aspektuelle Markierung für den Ausdruck der Simultanität in der Zielsprache verwenden. Dieses Ergebnis legt nahe, dass diese Lerner die grundlegende aspektuelle Opposition zwischen der perfektiven und der imperfektiven aspektuellen Form in der Zielsprache erkannt haben müssen. Da englische Probanden mit einer vergleichbaren Opposition aus ihrer Muttersprache ( \pm progressive Markierung) vertraut sind, können sie von diesem Wissen profitieren und dieses bereits sehr früh in der Zielsprache anwenden. Dieses spiegelt sich besonders deutlich in dem Gebrauch der stärkeren Aspektstrategie für den Simultanitätsausdruck bei Lernanfängern in L2 Tschechisch wider. Die Simultanität auf diese Weise zum Ausdruck zu bringen ist einfach, sehr effizient, informativ und auch zielsprachig.

In den Daten der deutschen Lernanfänger kommt explizite Aspektmarkierung kein einziges Mal vor. Diese Lerner verlassen sich ganz auf die Anwendung der adverbialen Strategie, wenn sie in der Zielsprache Simultanität ausdrücken. Analog zu der englischen Anfängergruppe ist das Vorgehen der deutschen Lernanfänger durch die Präferenzen der Ausgangssprache motiviert. Als Fazit lässt sich sagen, dass am Erwerbsanfang die englischen Lernanfänger einen Vorteil gegenüber den deutschen Lernanfängern haben, weil sie das zentrale Mittel der Zielsprache, den grammatischen Aspekt, vor dem Hintergrund ihrer muttersprachlichen Grammatik leichter identifizieren und für den Simultanitätsausdruck verwenden können.

Was passiert aber, wenn Lerner ein höheres Sprachniveau in der Zielsprache erreichen? Welche Rolle spielen dann die aus der L1 in die L2 übertragbaren Eigenschaften auf diesem Sprachniveau? 


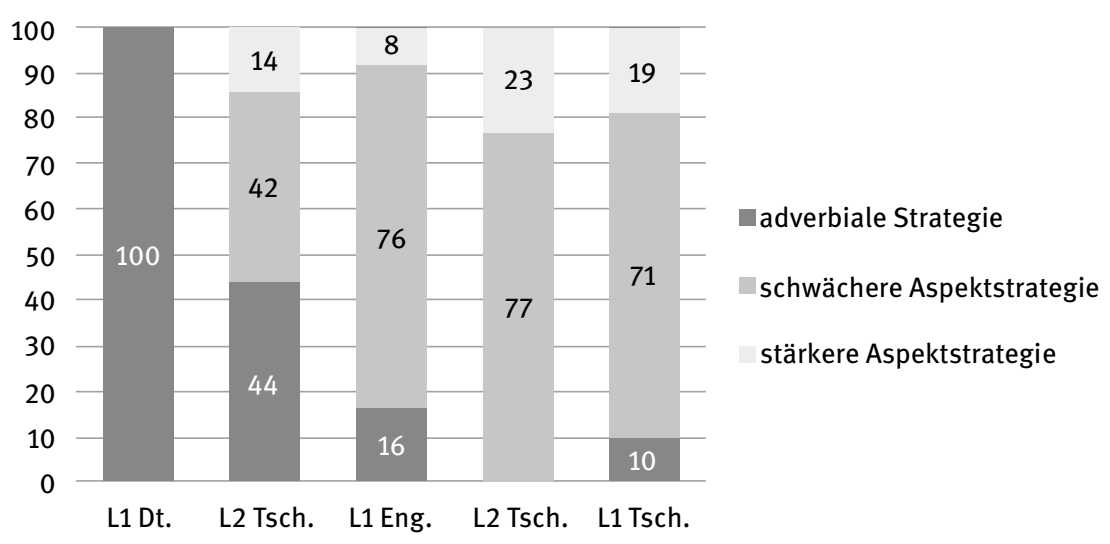

Abb. 35: Simultanitätsausdruck von englischen und deutschen fortgeschrittenen Lernern

Zwei interessante Beobachtungen lassen sich in Abbildung 35 bezüglich der englischen fortgeschrittenen Lerngruppe machen: 1) Sie gebrauchen häufiger als die Muttersprachler die explizite aspektuelle Markierung, wenn sie in L2 Tschechisch Simultanität ausdrücken (der so genannte overuse), und 2) entsprechend gebrauchen sie - aus der Perspektive der Zielsprache - die adverbiale Strategie nicht oft genug (der so genannte underuse). Auf dem Weg zur Zielsprache folgen die englischsprachigen Lerner weder dem zielsprachigen noch dem muttersprachlichen Muster. So entsteht eine Lernvarietät mit eigenen Gesetzmäßigkeiten.

Die deutschen fortgeschrittenen Lerner hingegen weisen einen ausgewogenen Trend auf: Sie setzten alle drei Strategien für den Simultanitätsausdruck ein, die in der Zielsprache zur Verfügung stehen. Somit nehmen sie das Muster auf, das auch für die tschechischen Muttersprachler typisch ist. Allgemein kann man sagen, dass deutsche Lerner in Bezug auf das Verteilungsmuster der drei Strategien der Zielsprache viel näherstehen, als es die englischen Lerner tun. Abbildung 36 präsentiert alle Sprachen - Lerner und Muttersprachler - im Zusammenhang mit den drei Strategien.

Aus Abbildung 36 wird ersichtlich, dass trotz der stärkeren Ähnlichkeit in der Art der Ausdrucksmittel und der Distribution der Strategien für den Simultanitätsausdruck zwischen L1 Tschechisch und L1 Englisch es gerade die deutschen und nicht die englischen fortgeschrittenen Lerner des Tschechischen sind, die dem zielsprachigen Muster am nächsten kommen. 


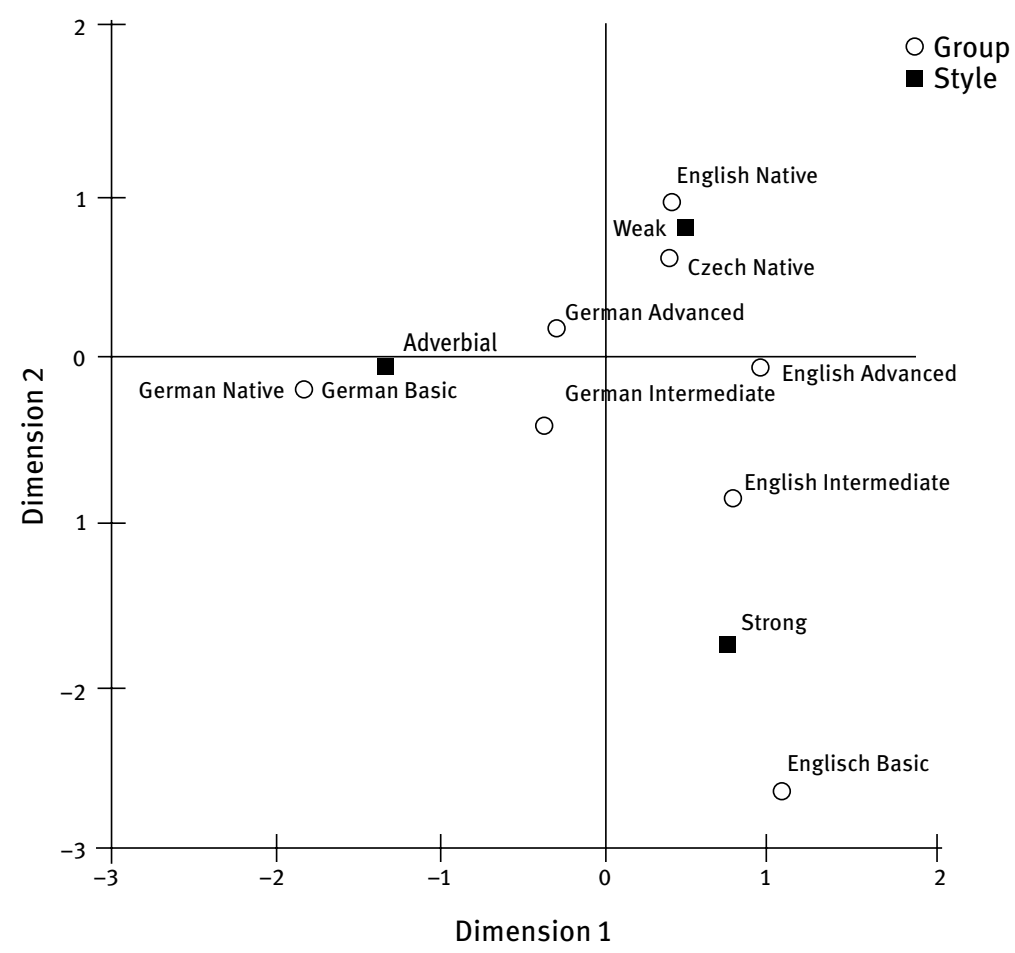

Abb. 36: Die so genannte 'centroids'-Darstellung für den Zusammenhang zwischen der verwendeten Strategie und der Sprechergruppe Tschechisch L1, Deutsch L1 und englische/ deutsche Lernergruppen L2 Tschechisch

\subsection{Fazit und Diskussion}

Das vorliegende Kapitel befasste sich mit der Frage, wie deutsche Lerner bei dem Erwerb von L2 Tschechisch vorgehen (Verlauf) und inwieweit sie sich dabei dem System der Zielsprache nähern können (Endzustand). Im Fokus standen dabei der Ausdruck der temporalen Simultanität und die Kategorie des grammatischen Aspekts.

Um diesen beiden Fragen besser auf den Grund gehen zu können, wurde eine Gruppe von englischen Lernern zum Vergleich herangezogen. Beide Lernergruppen waren hinsichtlich der Variablen wie sozioökonomischer Hintergrund, Alter und Bildung vergleichbar.

Es wurde gezeigt, dass für englische Lerner der Erwerb des Tschechischen dank der Übereinstimmungen zwischen der L1 und der L2 im Aspektbereich ein- 
facher ist. Dieses Bild ändert sich aber signifikant, wenn Lernerpräferenzen auf weiter fortgeschrittenen Sprachniveaus in Betracht gezogen werden. Der anfängliche Vorteil der englischen gegenüber den deutschen Lernanfängern geht verloren: Deutsche fortgeschrittene Lerner haben in der diskutierten Studie annähernd muttersprachliche Präferenzen für den Simultanitätsausdruck in der Zielsprache gelernt und sind somit der Zielsprache sehr nahe gekommen. Englische fortgeschrittene Lerner dagegen zeigten auf dem gleichen Sprachniveau klare Lernermuster.

Wie lassen sich diese Ergebnisse erklären? Es wäre plausibel anzunehmen, dass die aus der Ausgangssprache vertrauten Eigenschaften den Erwerb der Zielsprache nicht immer erleichtern oder beschleunigen, sondern diesen ab einem bestimmten Zeitpunkt erschweren bzw. verlangsamen. Es ist hier hervorzuheben, dass es in dem Prozess des Fremdsprachenlernens nicht nur um die Entdeckung und das Erlernen der Formen geht, sondern auch darum, dass die gelernten Formen angemessen (und das heißt zielsprachig) gebraucht werden. Dies gilt besonders für spätere Entwicklungsstadien des L2-Erwerbs und ist ausschlaggebend für die Diskussion über den Endzustand (fortgeschrittene und sehr fortgeschrittene Lerner). Die in diesem Kapitel vorgestellten Ergebnisse stehen im Einklang mit der Transfer- und Endzustand-Debatte (Dulay/Burt/Krashen 1982; Birdsong 1992; von Stutterheim 2003; Kap. 1 und 10).

Diese Hypothese wirft die folgende Frage auf: Ist es möglich, dass Lerner einer Fremdsprache durch die 'Ähnlichkeit' zwischen den Eigenschaften ihrer Muttersprache und der zu lernenden Zielsprache in die Irre geführt werden? Anhand der besprochenen Arbeit (Schmiedtová 2004) kann man diese Frage mit 'Ja' beantworten: Die englischen Lerner lassen sich insofern in die Irre führen, als sie ihre Anfangshypothese über die zielsprachige Vorgehensweise für den Ausdruck der Simultanität im Laufe des Erwerbs nicht weiter überprüfen bzw. an das tatsächliche Muster der Zielsprache anpassen.

Aus den präsentierten Daten der englischen Lerner geht hervor, dass sich die Präferenzen und ihre Verteilung zugunsten des zielsprachigen Musters auf der Zwischenstufe verändert zu haben scheinen. Was hier aber den Ausschlag gibt, ist, dass fortgeschrittene englische Lerner diesem in Richtung Zielsprache gehenden Trend nicht folgen. Im Allgemeinen heißt das, dass die Ähnlichkeiten zwischen der L1 und der L2 diese Lerngruppe daran gehindert haben, die Subtilitäten in L2 Tschechisch zu erlernen, die im Bereich der Simultanität das Englische und das Tschechische unterscheiden.

Die deutschen Lerner gingen nicht nur bei dem Erwerb der sprachlichen Mittel für den Simultanitätsausdruck anders vor, sondern sie haben auch einen anderen Endzustand als die englischen Lerner in der Zielsprache erlangt. Einer der Gründe dafür ist sicherlich die Tatsache, dass deutsche Lerner an die Ziel- 
sprache von Anfang an mit der Hypothese herangehen, die Zielsprache sei wesentlich anders als die Muttersprache. Diese Hypothese kommt allen, die das Tschechische oder eine andere slawische Sprache lernen oder gelernt haben, sicherlich bekannt vor. Wie beeinflusst aber diese Haltung den Erwerbsprozess?

Wie die Daten gezeigt haben, führt eine solche Hypothese $\mathrm{zu}$ Beginn des Erwerbs zunächst zu einem nicht-zielsprachigen Vorgehen. Man könnte sich auch vorstellen, dass der Erwerb unter diesen Umständen langsamer voranschreitet und auch das persönliche Empfinden über das Lerntempo und den -erfolg eher mäßig sind. Aber ab einem bestimmten Kenntnisstand ändert sich die Lage und der deutsche Lerner ist gerade wegen der fehlenden Übereinstimmungen zwischen L1 und L2 in der Lage, auch die subtilen Unterschiede in der Zielsprache $\mathrm{zu}$ identifizieren und zu lernen.

Die Ergebnisse der Studie von 2004 sind ermutigend: Entgegen der allgemeinen Meinung, die viele Deutsche teilen und die auch zum Teil in der Literatur vertreten wird, dass deutsche Muttersprachler eine slawische Sprache wie das Tschechische - insbesondere den Aspekt - kaum lernen können, zeigen unsere Daten, dass deutsche L1-Sprecher das Tschechische nicht nur gut erlernen können, sondern dabei im Endeffekt besser als die Vergleichsgruppe der englischen Muttersprachler abschneiden.

Neuere empirische Studien zum Erwerb von L2 Deutsch von tschechischen und russischen fortgeschrittenen Lernern (Schmiedtová/Sahonenko 2012; Kap. 8) haben zwei wichtige Ergebnisse erbracht: 1) Es konnte gezeigt werden, dass tschechische und russische Muttersprachler trotz der sehr starken Ähnlichkeit der zugrundeliegenden Aspektsysteme verschiedene Präferenzen im Aspektgebrauch haben. 2) Aus den Lernerdaten wurde klar, dass diese Präferenzen sich auch auf den Gebrauch der Zielsprache auswirken. Darüber hinaus konnte festgestellt werden, dass die tschechische Lerngruppe in L2 Deutsch 'muttersprachlicher' als es die russische Lerngruppe war. Dies hing nicht etwa damit zusammen, dass die tschechischen Sprecher in der Studie ein höheres Sprachniveau als die russischen Sprecher hatten, sondern vielmehr damit, dass in dem untersuchten Bereich die Ausgangssprachen Tschechisch/Deutsch erheblich mehr Gemeinsamkeiten als das Paar Russisch/Deutsch aufwiesen.

Diese Ergebnisse spiegeln sich auch in den informationsverarbeitenden Wahrnehmungsprozessen wider, die mit Hilfe von Augenbewegungsmessung von der psycholinguistischen Forschungsgruppe an der Universität Heidelberg untersucht wurden. Die Ergebnisse der Studien zeigen, dass in Verbindung mit sprachlichen Präferenzen auch visuelle Aufmerksamkeitsstrukturen variieren. Mit anderen Worten, die Muster des Blickbewegungsverhaltens bestätigen die in der sprachlichen Darstellung beobachteten Kontraste. 
Abschließend soll noch kurz auf den in der Einleitung erwähnten Sprachkontakt zwischen Deutsch und Tschechisch eingegangen werden. Bereits in Abschnitt 9.1 wurde festgestellt, dass der Einfluss des Deutschen über den Bereich des Lexikons hinausgeht und dass sich dieser sogar im Bereich der tschechischen Syntax niederschlägt. Es liegt nahe, dass der Einfluss des Deutschen noch tiefer greift und sich auch auf sprachliche Bereiche wie Informationsstruktur, Informationsselektion und Perspektivierung ausgewirkt hat. Dass in den Blickbewegungsstudien das Tschechische mit dem Deutschen und nicht mit dem Russischen eine Gruppe bildet, zeigt, dass sich das Deutsche und das Tschechische noch viel näherstehen, als bisher angenommen wurde.

\subsection{Zusammenfassung}

Kapitel 9 beschäftige sich mit dem Ausdruck der temporalen Simultanität in längeren Filmnacherzählungen von Muttersprachlern sowie Lernen des Tschechischen mit unterschiedlichem Beherrschungsgrad. Für den Ausdruck der Gleichzeitigkeit spielen aspektuelle Mittel eine wesentliche Rolle. Da das Englische im Unterschied zum Deutschen über ein Aspektsystem verfügt, das in bestimmten Merkmalen dem tschechischen Aspektsystem ähnelt, ist der Vergleich der beiden L2-Gruppen besonders interessant. Die Ergebnisse zeigen, dass zu Erwerbsbeginn aufgrund der Ähnlichkeiten des englischen und tschechischen Aspektsystems die englischen Lerner einen Vorteil gegenüber den deutschen Lernern haben. Später im Erwerbsprozess jedoch kommen deutsche Sprecher viel näher an das Aspektsystem der Zielsprache als englische Lerner. Diese Befunde werfen neue Fragen im Hinblick auf die kontaktlinguistischen Zusammenhänge zwischen dem Deutschen und dem Tschechischen auf. 
